\title{
Does Beam Angle Optimization Really Matter for Intensity-Modulated Radiation Therapy?
}

\author{
Humberto Rocha ${ }^{1}$, Joana M. Dias ${ }^{1,2}$, Brigida C. Ferreira ${ }^{3,4}$, and \\ Maria do Carmo Lopes ${ }^{3,4}$ \\ 1 INESC-Coimbra, Rua Antero de Quental, 199 \\ 3000-033 Coimbra, Portugal \\ 2 Faculdade de Economia, Universidade de Coimbra, \\ 3004-512 Coimbra, Portugal \\ 3 School for Allied Health Technologies, \\ 4400-330 Porto, Portugal \\ 4 Serviço de Física Médica, IPOC-FG, EPE, \\ 3000-075 Coimbra, Portugal \\ hrocha@mat.uc.pt, joana@fe.uc.pt, bcf@estsp.ipp.pt, \\ mclopes@ipocoimbra.min-saude.pt
}

\begin{abstract}
The beam angle optimization (BAO) in intensity-modulated radiation therapy (IMRT) consists on the selection of appropriate radiation incidence directions and can theoretically influence the quality of the IMRT plans, both by improving tumor coverage and by obtaining better organs sparing. However, in clinical practice, the importance of $\mathrm{BAO}$ is yet to be acknowledged and, most of the time, beam directions continue to be either equispaced or manually selected by the treatment planner, not making integral part of the optimization loop that is typically devoted to the fluences optimization. During this optimization loop, the treatment planner needs to tune different parameters, in a time consuming process, including, most of the time, objective function weights associated to the different structures included in the optimization procedure. Traditionally, the treatment planning is seen as a sequential process that starts with the selection of the irradiation beam directions. When irradiation beam directions are selected through a BAO procedure that uses the optimal values of the fluence optimization (for a given set of initial objective function weight parameters), does the posterior tuning of the objective function parameters completely jeopardize the BAO effort? The goal of this paper is to contribute to clarify this question, to perceive the importance of BAO in IMRT and its timing within the treatment planning optimization loop. For a study set of ten clinical cases of head-and-neck tumors treated at the Portuguese Institute of Oncology of Coimbra we showed that optimized beam angle sets obtained statistical significant better results $(p-$ value $<0.001)$ than the traditional equispaced configuration. Furthermore, in our tests, despite optimal beam angle sets being always preferable to equispaced configurations, the choice of the weight parameters for angle selection showed influence on the correspondent plan quality.
\end{abstract}

Keywords: Radiotherapy; IMRT; Beam angle optimization; Direct Search 


\section{Introduction}

Intensity-modulated radiation therapy (IMRT) is a modern technique with a treatment planning that is usually a sequential process where initially a given number of radiation incidence directions are selected followed by the optimization of the radiation intensities considering those irradiation directions. The selection of appropriate irradiation directions in IMRT treatment planning - beam angle optimization (BAO) problem - is the first problem that arises in treatment planning, but its optimal solution is highly dependent on the optimal solution of the fluence map optimization (FMO) problem - the problem of deciding what are the optimal radiation intensities associated with each set of beam angles. When the $\mathrm{BAO}$ problem is not based on the optimal FMO solutions, the resulting beam angle set has no guarantee of optimality and has questionable reliability since it has been extensively reported that optimal beam angles for IMRT are often non-intuitive [20]. Nevertheless, in current clinical practice, most of the time, beam directions are considered equispaced or continue to be manually selected which requires many trial and error iterations between selecting beam angles and computing fluence patterns until a suitable treatment is achieved. Therefore, regardless the evidence presented in the literature that appropriate irradiation directions can lead to a plan's quality improvement $[2,10,21], \mathrm{BAO}$ continue to be either ignored or not making integral part of the treatment planning optimization loop that is typically dominated by the FMO problem.

IMRT treatment planning generally has to balance a multitude of risks and goals that should be, ideally, addressed by multiobjective approaches to achieve acceptable compromises defined a priori or selected a posteriori from a database of Pareto-optimal generated plans. Recently, a posteriori [6] and a priori [4] multi-criteria approaches have been proposed for IMRT. However, the inclusion of a full BAO integrated into a fluence map multiobjective optimization framework has still many underlying issues including clinically acceptable computational times. Moreover, the approaches proposed in most of the literature and offered by the vast majority of the treatment planning systems (TPS) treatment plan dedicated commercial software applications - consider weighted mathematical formulations to model the FMO problem and balance the multiple conflicting objectives (e.g., achieve a high tumor dose while giving a low dose to neighboring organs). In current clinical practice, this balance is attempted by a treatment planner trying to steer the TPS by interactively tuning plan parameters towards a better solution. For each patient, the planner makes a first choice for the different parameters (including the weights assigned to each structure incorporated in the optimization loop) based on local protocols or experience. The TPS then generates a treatment plan with corresponding dose distribution. If the dose distribution is not satisfactory, then the planner tune the parameters (based on experience) for a new run of the algorithm until the dose distribution is considered satisfactory or the time to further improve it runs out. Typically, this parameter tuning (often called "optimization") is done for a fixed set of beam radiation incidence directions that are either chosen by the planner or are obtained after an initial BAO procedure. As referred above, the optimal solution 
of the BAO should depend on the optimal solution of the FMO problem. However, the optimal incidence radiation directions are obtained using a given set of parameters, in particular a given set of objective function weights associated to the different structures included in the optimization procedure. Afterwards, does the tuning of the fluence map objective function parameters completely jeopardize the initial BAO effort? Is there a timing to perform BAO in order to diminish deterioration of $\mathrm{BAO}$ results? The goal of this paper is to contribute to clarify this questions, to perceive the importance of BAO in IMRT and its timing within the treatment planning optimization loop.

\section{Methods and Materials}

\subsection{Study Patients}

Ten clinical examples of head-and-neck tumors treated at the Portuguese Institute of Oncology of Coimbra (IPOC), signalized as complex cases where proper target coverage and organ sparing, in particular parotid sparing, proved to be difficult to obtain with the typical 7-beam equispaced coplanar treatment plans, were selected. The patients' computed tomography (CT) sets and delineated structures were exported via Dicom RT to a freeware computational environment for radiotherapy research (CERR).

In general, the head-and-neck region is a complex area to treat with radiotherapy due to the large number of sensitive organs in this region (e.g., eyes, mandible, larynx, oral cavity, etc.). The spinal cord and the brainstem are some of the most critical organs at risk (OARs) in the head-and-neck tumor cases. These are serial type organs, i.e., organs such that if only one functional subunit is damaged, the whole organ functionality is compromised. Therefore, if the tolerance dose is exceeded, it may result in functional damage to the whole organ. Thus, it is extremely important not to exceed the tolerance dose assigned for these type of organs. Other than the spinal cord and the brainstem, the parotid glands are also important OARs. The parotid gland is the largest of the three salivary glands. A common complication due to parotid glands irradiation is xerostomia. This secondary radiation effect decreases the quality of life of patients undergoing radiation therapy of head-and-neck, causing difficulties to swallow. The parotids are parallel organs, i.e., if a small volume of the organ is damaged, the organ functionality may not be affected. Their tolerance dose depends strongly on the fraction of the volume irradiated. Hence, if only a small fraction of the organ is irradiated the tolerance dose is much higher than if a larger fraction is irradiated. Thus, for these parallel type structures, the organ mean dose is generally used as an objective for inverse planning optimization. In this retrospective study, the OARs used for treatment optimization were limited to the spinal cord, the brainstem and the parotid glands.

For the head-and-neck cases in study the planning target volume (PTV) consisted of $P T V_{70}$ and $P T V_{59.4}$ corresponding to different prescribed doses. The prescription dose for the target volumes and tolerance doses for the OARs considered in the optimization are presented in Table 1. 
Table 1. Tolerance and prescribed doses for all the structures considered for IMRT optimization.

\begin{tabular}{lccc}
\hline Structure & Mean dose & Max dose Prescribed dose \\
\hline Spinal cord & - & $45 \mathrm{~Gy}$ & - \\
Brainstem & - & $54 \mathrm{~Gy}$ & - \\
Left parotid & $26 \mathrm{~Gy}$ & - & - \\
Right parotid & $26 \mathrm{~Gy}$ & - & - \\
PTV $_{\mathbf{7 0}}$ & - & - & $70.0 \mathrm{~Gy}$ \\
PTV $_{\mathbf{5 9 . 4}}$ & - & - & $59.4 \mathrm{~Gy}$ \\
\hline
\end{tabular}

\section{$2.2 \quad$ FMO Model}

For optimization purposes, radiation dose distribution deposited in the patient, measured in Gray (Gy), needs to be assessed accurately. Each structure's volume is discretized in voxels (small volume elements) and the dose is computed for each voxel using the superposition principle, i.e., considering the contribution of each beamlet. Typically, a dose matrix D is constructed from the collection of all beamlet weights, by indexing the rows of $\mathrm{D}$ to each voxel and the columns to each beamlet, i.e., the number of rows of matrix $\mathrm{D}$ equals the number of voxels $(V)$ and the number of columns equals the number of beamlets $(N)$ from all beam directions considered. Therefore, using matrix format, we can say that the total dose received by the voxel $i$ is given by $\sum_{j=1}^{N} D_{i j} w_{j}$, with $w_{j}$ the weight of beamlet $j$. Usually, the total number of voxels considered reaches the tens of thousands, thus the row dimension of the dose matrix is of that magnitude. The size of $D$ originates large-scale problems being one of the main reasons for the difficulty of solving the FMO problem.

Many mathematical optimization models and algorithms have been proposed for the FMO problem, including linear models [18], mixed integer linear models [9], nonlinear models [5], and multi-criteria models [3]. Most of the FMO models in the literature belong to a class of constrained optimization models such that an objective function is optimized while meeting dose requirements. A variety of criteria may be considered to be included in the objective function, leading to many different objective functions. It is beyond the scope of this study to discuss which formulation of the FMO problem is preferable. Romeijn et al. [19] demonstrated that most of the treatment plan evaluation criteria proposed in the medical physics literature are equivalent to convex penalty function criteria when viewed as a multicriteria optimization problem. Here, we will use a convex penalty function voxel-based nonlinear model [1]. The conclusions drawn regarding this particular model are valid also if different weighted mathematical formulations to model the FMO problem are considered. In this model, each voxel is penalized according to the square difference of the amount of dose received by the voxel and the amount of dose desired/allowed for the voxel. This formulation yields a quadratic programming problem with only linear non-negativity 
constraints on the fluence values [18]:

$$
\begin{aligned}
& \min _{w}\left[\underline{\lambda}_{i}\left(T_{i}-\sum_{j=1}^{N} D_{i j} w_{j}\right)_{+}^{2}+\bar{\lambda}_{i}\left(\sum_{j=1}^{N} D_{i j} w_{j}-T_{i}\right)_{+}^{2}\right] \\
& \text { s.t. } \quad 0 \leq w_{j} \leq w^{\text {max }}, j=1, \ldots, N
\end{aligned}
$$

where $T_{i}$ is the desired dose for voxel $i, \underline{\lambda}_{i}$ and $\bar{\lambda}_{i}$ are the penalty weights of underdose and overdose of voxel $i, w^{\max }$ is the maximum beamlet intensity allowed and $(\cdot)_{+}=\max \{0, \cdot\}$. This nonlinear formulation implies that a very small amount of underdose or overdose may be accepted in clinical decision making, but larger deviations from the desired/allowed doses are decreasingly tolerated [1].

The optimal solutions obtained ensure that the resulting treatment is the best possible with respect to the weighting parameters $(\lambda)$ used. Since it is impossible to attribute effective clinical meaning to the weight parameters, the 'optimal' weighting scheme is unknown and the choice of the weights is typically a long trial-and-error process until a satisfactory solution is achieved. Furthermore, for beam angle optimization it is not clear how traditional trial-and-error parameter tuning should be incorporated or managed.

\subsection{BAO Approach}

In order to model the $\mathrm{BAO}$ problem as a mathematical programming problem, a quantitative measure to compare the quality of different sets of beam angles is required. For the reasons presented before, our approach for modelling the $\mathrm{BAO}$ problem uses the optimal solution value of the FMO problem as measure of the quality of a given beam angle set. Many authors consider non-coplanar angles $[2,4,7,11,12,13]$ which result in potentially improved treatment plans [14,22]. However, despite the fact that almost every angle is possible for radiation delivery, the use of coplanar angles is predominant. For simplicity, only coplanar angles will be considered.

Let us consider $n$ to be the fixed number of (coplanar) beam directions, i.e., $n$ beam angles are chosen on a circle around the CT-slice of the body that contains the isocenter (usually the center of mass of the tumor). In our formulation we consider all continuous $\left[0^{\circ}, 360^{\circ}\right]$ gantry angles instead of a discretized sample. Since for $\alpha, \beta \in\left[0^{\circ}, 360^{\circ}\right]$, the angle $360^{\circ}+\alpha$ is the same as the angle $\alpha \in$ $\left[0^{\circ}, 360^{\circ}\right]$ and the angle $-\beta$ is equivalent to the angle $360^{\circ}-\beta \in\left[0^{\circ}, 360^{\circ}\right]$, we can avoid a bounded formulation. A basic formulation for the BAO problem is obtained by selecting an objective function such that the best set of beam angles is obtained for the function's minimum:

$$
\begin{aligned}
& \min f\left(\theta_{1}, \ldots, \theta_{n}\right) \\
& \text { s.t. } \theta_{1}, \ldots, \theta_{n} \in \mathbb{R}^{n} .
\end{aligned}
$$


Here, the objective $f\left(\theta_{1}, \ldots, \theta_{n}\right)$ that measures the quality of the set of beam directions $\theta_{1}, \ldots, \theta_{n}$ is the optimal value of the FMO problem for each fixed set of beam directions.

This BAO formulation facilitates the use of pattern search methods (PSM). PSM are derivative-free optimization algorithms that require few function evaluations to progress and converge and have the ability to better avoid local entrapment making them a suitable approach for the resolution of the highly non-convex BAO problem $[15,16,17]$. PSM are directional search methods that use positive bases to move in a direction that produces a decrease in the objective function. The main feature of a positive basis, that motivates PSM, is that for any given vector, in particular for the gradient vector, there is a vector of the positive basis that forms an acute angle with the gradient vector which means that it is a descent direction. PSM are organized around two phases at every iteration: one that assures convergence to a local minimum (poll), and the other (search) where flexibility is conferred to the method allowing searches away from the neighborhood of the current iterate. PSM are used to address the $\mathrm{BAO}$ problem.

\subsection{Computational Tests}

Our tests were performed on a 2.66Ghz Intel Core Duo PC with 3 GB RAM. The computational tools developed within MATLAB and CERR - computational environment for radiotherapy research [8] - were used to obtain the dosimetric data input for treatment plan optimization and also to facilitate convenient access, visualization and analysis of patient treatment planning data. To address the convex nonlinear formulation of the FMO problem we used a trustregion-reflective algorithm (fmincon) of MATLAB 7.4.0 (R2007a) Optimization Toolbox. A tailored version of pattern search methods that include beams-eyeview dose metrics in the search step so that directions with larger dose metric scores are tested first improving results and computational time [16] was used to tackle the BAO problem.

The FMO problem is inherently a multicriteria optimization problem with conflicting objectives. Despite the convex nonlinear formulation being commonly used for FMO and $\mathrm{BAO}$, it requires the subjective decision of assigning penalty weights to be used, which is an handicap. A set of parameter that produces acceptable treatment plans for the equispaced beam angle configuration [16] corresponds to assign $\underline{\lambda}_{i}=\bar{\lambda}_{i}=4$ to the target volumes and $\bar{\lambda}_{i}=2$ to the OARs and remaining tissue. For this set of penalty weight parameters, an optimal beam angle set was obtained for each patient using our BAO approach and denoted $B A O$. For a unitary set of penalty weight parameters, i.e. considering the previous parameters equal to one, an optimal beam angle set was also obtained for each patient using our BAO approach and denoted BAO1. For each of the 10 patients, three different 7-beam angle configurations - BAO, BAO1 and the equispaced configuration denoted Equi - were tested considering 100 different fluence map objective function weight parameters selected randomly 
Table 2. Mean values and corresponding $p$-value of the one-way Anova for the structure metrics considered in IMRT optimization.

\begin{tabular}{|c|c|c|c|c|}
\hline Structure (metric) & Equi & $B A O$ & $B A O 1$ & $p-$ value \\
\hline Spinal cord $\left(D_{\max }\right)$ & 40.6015 & Gy 40.1501 & Gy 39.7543 Gy & $<0.001$ \\
\hline Left parotid $\left(D_{\text {mean }}\right)$ & 26.2670 & Gy 25.3533 & Gy 26.1470 Gy & $<0.001$ \\
\hline Right parotid $\left(D_{\text {mean }}\right)$ & 26.1721 & Gy 24.6396 & Gy 25.8298 Gy & $<0.001$ \\
\hline Brainstem $\left(D_{\max }\right)$ & 50.4473 & Gy 49.5464 & Gy 49.9163 Gy & $<0.001$ \\
\hline $\mathbf{P T V}_{\mathbf{7 0}}\left(D_{95 \%}\right)$ & 66.6182 & Gy 66.6899 & Gy 66.6643 Gy & 0.5017 \\
\hline $\mathbf{P T V}_{59.4}\left(D_{95 \%}\right)$ & 57.0359 & Gy 57.2516 & Gy 57.1582 Gy & 0.0242 \\
\hline
\end{tabular}

in an automated way in the interval $[2,8]$ for tumors and $[1,4]$ for OARS and remaining tissue.

\section{Results}

Treatment plans are typically compared by judging their cumulative dosevolume histograms (DVHs) and/or by comparing different metrics that usually include the dose delivered to $95 \%$ of the tumor $\left(D_{95 \%}\right)$ and the mean $\left(D_{\text {mean }}\right)$ or maximum doses $\left(D_{\max }\right)$ of OARs. Boxplots of the metric values considering the 100 treatment plans corresponding to the different penalty weight parameters for each of the 10 patients are displayed in Fig. 1 comparing the performance of the three beam angle sets. By simple inspection we can observe that organ sparing metrics are better for the optimized beam angle sets, in particular for $B A O$, for similar target coverage metric values.

One-way Anova (using MATLAB Statistics toolbox) was used to compare the means of the aggregated metric results of the three beam angle sets, to test the hypothesis that they are all the same, against the general alternative that they are not all the same. The statistical results are presented in Table 2 and we can confirm that metric means are different for all structures with statistic significance except for PTV70.

Since we are comparing more than two sets of beam angles, we need further information about which pairs of means are significantly different, and which are not. A multiple comparison procedure using the Tukey's honestly significant difference criterion was performed (post-hoc test available in MATLAB Statistics toolbox) and the output is displayed in Fig. 2. We can observe that there are no significant differences for the PTV70 metric but for the remaining structures, the optimized beam angle set obtained always better results with statistical significance $(p-$ value $<0.001)$. Furthermore, the optimized beam angle set $B A O$, obtained with weight parameters that lead to acceptable treatment plans for the equispaced configuration, clearly presented the overall better results.

We should note that mean results must be judged carefully and their main purpose is to compare overall results. For instance, mean results for the left parotid displayed in Table 2 show that BAO obtained an average sparing of 
1.5 Gy compared to equispaced configurations for the 10 patients. However, for some patients the improvement is residual while for others is important (up to 6 Gy) - see Fig. 2. In an ideal forthcoming automated treatment planing, where computational time and effort are less limitative, results should be evaluated case by case and if $\mathrm{BAO}$ makes a strong impact in a single patient being better in overall average, that should prove its importance.

\section{Discussion and Conclusions}

The benefits of BAO are yet to be fully acknowledged in clinical practice. Regardless the evidence presented in the literature that appropriate radiation beam incidence directions can theoretically lead to a plan's quality improvement, in practice, the choice of beam angles is, most of the time, simply regarded as just another set of parameters that need to be selected and whose update can lead to the exact same unpredictable result as changing the weight parameters of many TPS weighted mathematical formulation to model the FMO problem. Updating beam incidence directions is even more risky for IMRT since it has been extensively reported that optimal beam angles for IMRT are often non-intuitive.

This study aims to be a contribute for the perception of the interest of BAO in IMRT by learning if the update of the fluence map objective function parameters jeopardize an initial BAO effort and if there is a timing to perform $\mathrm{BAO}$ in order to diminish potential result's deterioration. For our study set of ten clinical cases of head-and-neck tumors treated at the Portuguese Institute of Oncology of Coimbra we showed that optimized beam angle sets obtained statistical significant better results than the traditional equispaced configuration in terms of target coverage and organ sparing metrics. Furthermore, in our tests, despite optimal beam angle sets being always preferable to equispaced configurations, the choice of the weight parameters for angle selection showed influence on the correspondent plan quality. Therefore, the first result of this study lead to the conclusion that optimized beam angles can lead to better treatment plans than the traditional equispaced beam angles. The second result indicate that BAO should be performed only when a set of parameters that lead to an acceptable treatment plan for the equispaced beam angle configuration is found. These conclusions fully answer the questions raised in the introduction of the paper/study.

Finally, this study further validates our BAO approach. To the best of our knowledge, this is the first study that compares different beam angle sets using many treatment plans corresponding to different FMO weight parameters. When comparing optimized beam angle sets with the equispaced beam angle configuration using a single set of FMO weight parameters, the conclusions can be biased by the fact that larger weight parameters may lead the BAO procedure to obtain better results for the corresponding structures. Therefore, this study also contributes to a fair comparison of beam angle sets when FMO problem is a weighted mathematical formulation. 

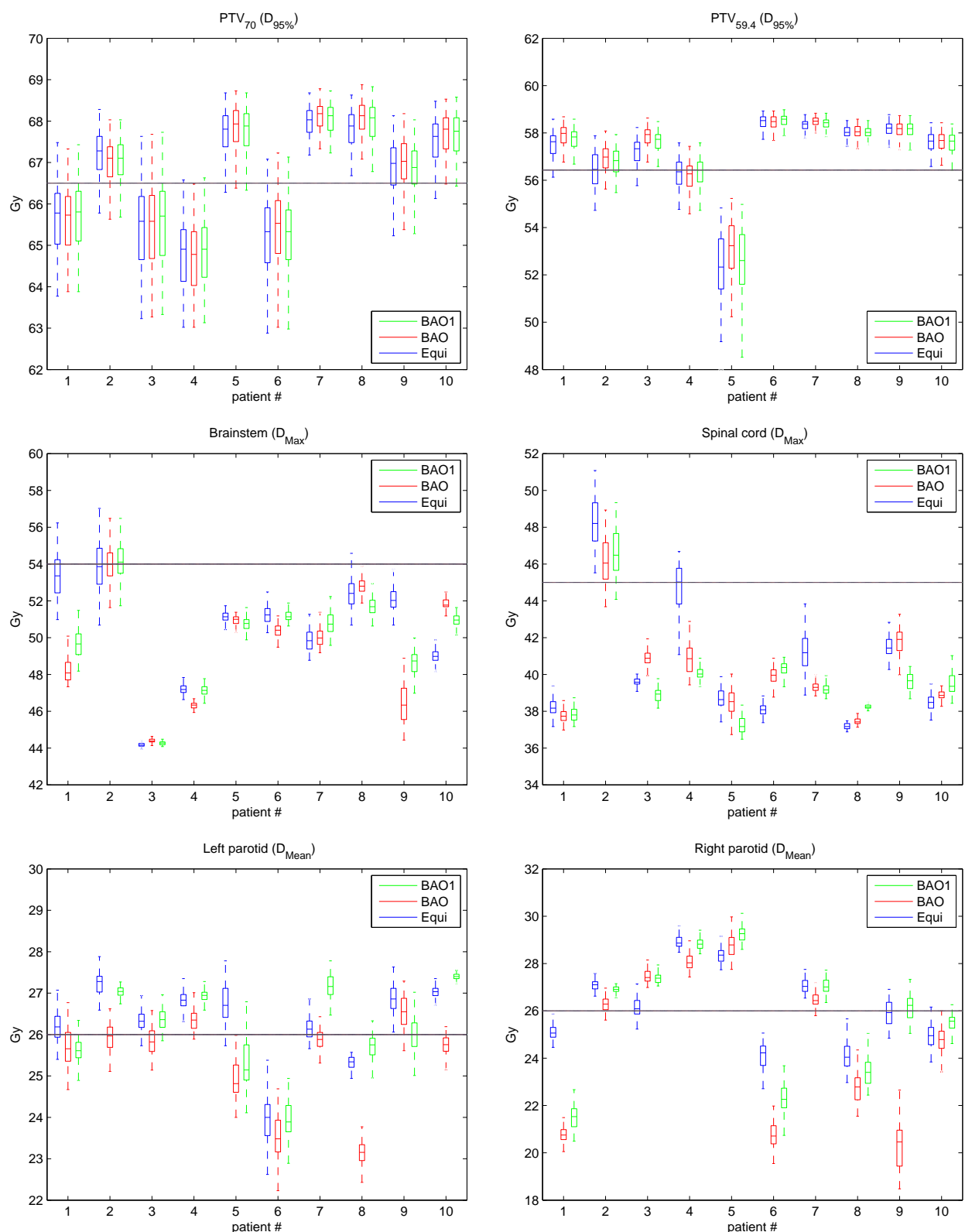

Fig. 1. Target coverage and organ sparing metric values of 100 treatment plans for each patient and for the three beam angle configurations tested: Equi, BAO and BAO1

\section{Acknowledgements}

This work was supported by FEDER. COMPETE. iCIS (CENTRO-07-ST24FEDER-002003). Portuguese Foundation for Science and Technology under project grants UID/MULTI/00308/2013 and PTDC/EIA-CCO/121450/2010. 

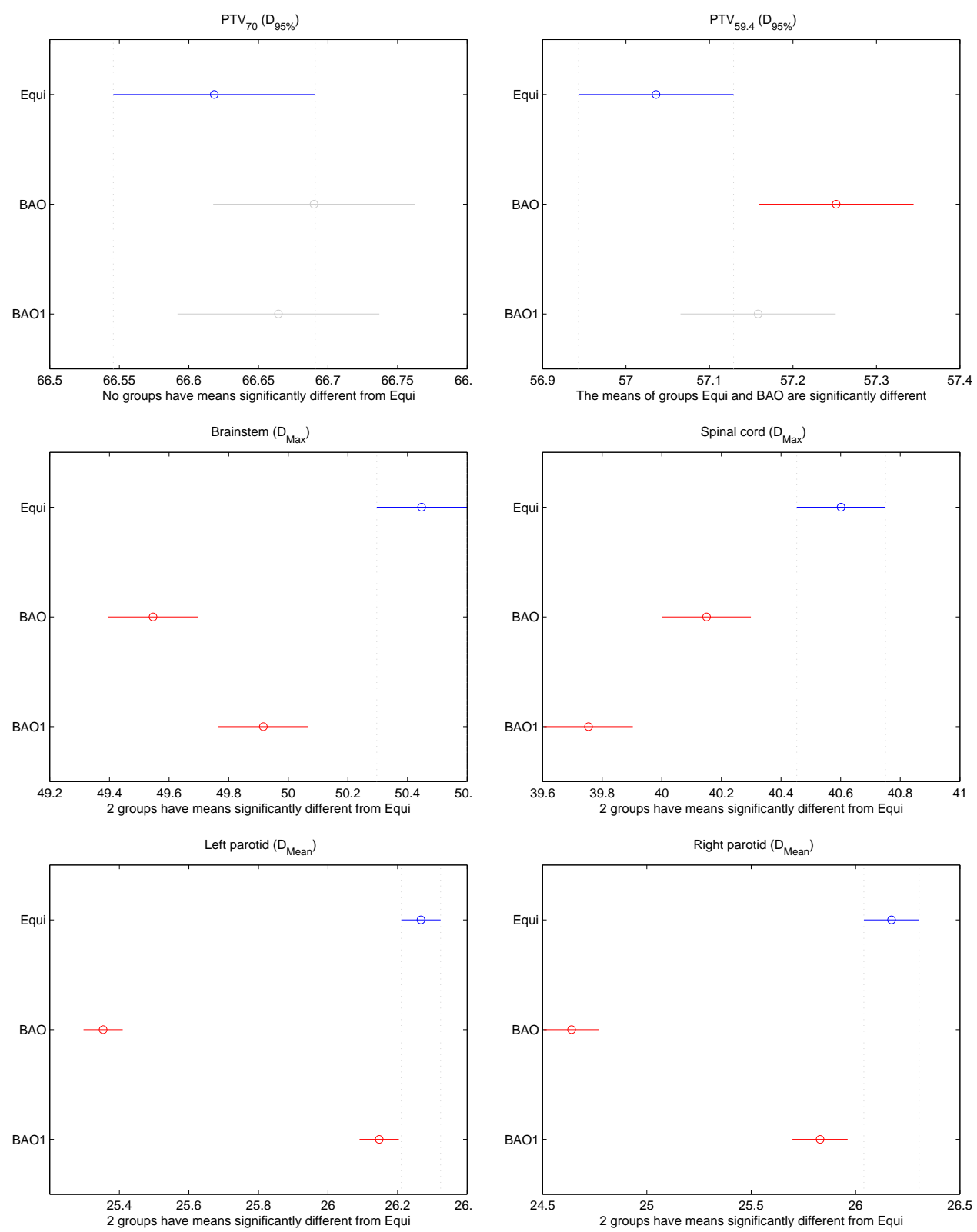

Fig. 2. Multiple comparison interactive graph of the overall target coverage and organ sparing metrics mean and confidence interval differences between the three beam angle configurations tested: Equi, BAO and BAO1 


\section{References}

1. Aleman, D.M., Kumar, A., Ahuja, R.K., Romeijn, H.E., Dempsey, J.F.: Neighborhood search approaches to beam orientation optimization in intensity modulated radiation therapy treatment planning. J. Global Optim. 42, 587-607 (2008)

2. Aleman, D.M., Romeijn, H.E., Dempsey, J.F.: A response surface approach to beam orientation optimization in intensity modulated radiation therapy treatment planning. INFORMS J. Comput.: Computat. Biol. Med. Appl. 21, 62-76 (2009)

3. Breedveld, S., Storchi, P.R.M., Keijzer, M., Heemink, A.W., Heijmen, B.J.M.: A novel approach to multi-criteria inverse planning for IMRT. Phys. Med. Biol. 52, 6339-6353 (2007)

4. Breedveld, S., Storchi, P., Voet, P., Heijmen, B.: iCycle: integrated, multicriterial beam angle, and profile optimization for generation of coplanar and noncoplanar IMRT plans. Med. Phys. 39, 951-963 (2012)

5. Cheong, K., Suh, T., Romeijn, H., Li, J., Dempsey, J.: Fast Nonlinear Optimization with Simple Bounds for IMRT Planning. Med. Phys. 32, 1975 (2005)

6. Craft, D., Monz, M.: Simultaneous navigation of multiple Pareto surfaces, with an application to multicriteria IMRT planning with multiple beam angle configurations. Med. Phys. 37, 736-741 (2010)

7. Das, S.K., Marks, L.B.: Selection of coplanar or non coplanar beams using threedimensional optimization based on maximum beam separation and minimized nontarget irradiation. Int. J. Radiat. Oncol. Biol. Phys. 38, 643-655 (1997)

8. Deasy, J.O., Blanco, A.I., Clark, V.H.: CERR: A Computational Environment for Radiotherapy Research. Med. Phys. 30, 979-985 (2003)

9. Lee, E.K., Fox, T., Crocker, I.: Integer programming applied to intensity-modulated radiation therapy treatment planning. Ann. Oper. Res. 119, 165-181 (2003)

10. Liu, H.H., Jauregui, M., Zhang, X., Wang, X., Dongand, L., Mohan, R.: Beam angle optimization and reduction for intensity-modulated radiation therapy of nonsmall-cell lung cancers. Int. J. Radiat. Oncol. Biol. Phys. 65, 561-572 (2006)

11. Lu, H.M., Kooy, H.M., Leber, Z.H., Ledoux, R.J.: Optimized beam planning for linear accelerator-based stereotactic radiosurgery. Int. J. Radiat. Oncol. Biol. Phys. 39, 1183-1189 (1997)

12. Meedt, G., Alber, M., Nüsslin, F.: Non-coplanar beam direction optimization for intensity-modulated radiotherapy. Phys. Med. Biol. 48, 2999-3019 (2003)

13. Mišić, V.V., Aleman, D.M., Sharpe, M.B.: Neighborhood search approaches to noncoplanar beam orientation optimization for total marrow irradiation using IMRT. Eur. J. Oper. Res. 205, 522-527 (2010)

14. Pooter, J.A., Romero, A.M., Jansen, W.P.A., Storchi, P.R.M., Woudstra, E., Levendag, P.C., Heijmen, B.J.M.: Computer optimization of noncoplanar beam setups improves stereotactic treatment of liver tumors. Int. J. Radiat. Oncol. Biol. Phys. 66, 913-922 (2006)

15. Rocha, H., Dias, J.M., Ferreira, B.C., Lopes, M.C.: Selection of intensity modulated radiation therapy treatment beam directions using radial basis functions within a pattern search methods framework. J. Glob. Optim. 57, 1065-1089 (2013)

16. Rocha, H., Dias, J.M., Ferreira, B.C., Lopes, M.C.: Beam angle optimization for intensity-modulated radiation therapy using a guided pattern search method. Phys. Med. Biol. 58, 2939-2953 (2013)

17. Rocha, H., Dias, J.M., Ferreira, B.C., Lopes, M.C.: Pattern search methods framework for beam angle optimization in radiotherapy design. Appl. Math. Comput. 219, 10853-10865 (2013) 
18. Romeijn, H.E., Ahuja, R.K., Dempsey, J.F., Kumar, A., Li, J.: A novel linear programming approach to fluence map optimization for intensity modulated radiation therapy treatment planing. Phys. Med. Biol. 48, 3521-3542 (2003)

19. Romeijn, H.E., Dempsey, J.F., Li, J.: A unifying framework for multi-criteria fluence map optimization models. Phys. Med. Biol. 49, 1991-2013 (2004)

20. Stein, J., Mohan, R., Wang, X.H., Bortfeld, T., Wu, Q., Preiser, K., Ling, C.C., Schlegel, W.: Number and orientation of beams in intensity-modulated radiation treatments. Med. Phys. 24, 149-160 (1997)

21. Voet, P., Breedveld, S., Dirkx, M., Levendag, P., Heijmen, B.: Integrated multicriterial optimization of beam angles and intensity profiles for coplanar and noncoplanar head and neck IMRT and implications for VMAT. Med. Phys. 39, 4858 (2012)

22. Wang, X., Zhang, X., Dong, L., Liu, H., Gillin, M., Ahmad, A., Ang, K., Mohan, R.: Effectiveness of noncoplanar IMRT planning using a parallelized multiresolution beam angle optimization method for paranasal sinus carcinoma. Int. J. Radiat. Oncol. Biol. Phys. 63, 594-601 (2005) 